Received: 20.06 .2021

Revised: 20.08 .2021

Accepted: 27.08 .2021

DOI: $10.17804 / 2410-9908.2021 .4 .006-014$

\title{
IN-SITU NUCLEAR REACTION ANALYSIS
}

\author{
V. B. Vykhodets ${ }^{\text {a) }}$, T. E. Kurennykh ${ }^{\text {b)* }}$ \\ M.N. Miheev Institute of Metal Physics, Ural Branch of the Russian Academy of Sciences, \\ 18, S. Kovalevskoy St., Ekaterinburg, 620137, Russian Federation \\ a) (iD http://orcid.org/0000-0001-9522-9147; \\ b) (iD http://orcid.org/0000-0001-9859-3374

\begin{abstract}
*Corresponding author: E-mail: kurennykh@imp.uran.ru
Address for correspondence: 18, S. ul. Kovalevskoy, Ekaterinburg, 620990, Ekaterinburg, Russian Federation Tel.: +7 (343) 3783846
\end{abstract}

The principles of in-situ nuclear reaction techniques and the need for them in various fields of scientific research are considered; several examples of the application of these techniques are given. It is shown that the techniques of in-situ nuclear reactions are effective in studying the diffusion of deuterium in metals at temperatures below room temperature, the diffusion of deuterium in proton-conducting oxides, the quantum diffusion of deuterium in metals at cryogenic temperatures, and the chemical composition of oxide nanopowders when they are heated in vacuum. Promising applications of nuclear reaction techniques in situ are formulated.

Keywords: nuclear reaction techniques, deuterium, metal, oxide, nanopowder.

\section{Acknowledgment}

The research was carried out within the state assignment from the Ministry of Science and Higher Education of the Russian Federation (theme "Function" No. AAAA-A19-119012990095-0).

\section{References}

1. The Stopping and Ranges of Ions in Matter (SRIM-2013): collection of software packages. Available at: http://www.srim.org.

2. Trakhtenberg I.Sh., Vladimirov A.B., Rubstein A.P. Ziegler J.F., Biersack J.P., Littmark U. The Stopping and Ranges of Ions in Solids, Pergamon Press, N.Y., 1984, vol. 1.

3. Rubshtein A.P., Trakhtenberg I., Volkova E., Vladimirov A.B., Gontar A., Uemura K. The interrelation between structure and mechanical properties of $\mathrm{CN} x$ films $(0 \leq \mathrm{x} \leq 0.5)$, deposited by arc sputtering of graphite. Diamond and Related Materials, 2005, vol. 14, pp. 1820-1823. DOI: 10.1016/J.DIAMOND.2005.07.016.

4. Trakhtenberg I.Sh., Vladimirov A.B., Rubstein A.P., Yugov V.A., Vykhodets V.B., Kurennykh T.E., Gontar A.G., Tkach V.N., Dub S.N., and Uemura K. Mechanical properties of CN $x$ coatings obtained by carbon arc sputtering. Journal of Superhard Materials, 2007, vol. 29, No. 3, pp. 138-141. DOI: 10.3103/S1063457607030045.

5. Rubstein A.P., Makarova E.B., Trakhtenberg I.Sh., Kudryavtseva I.P., Bliznets D.G., Philippov Yu.I., Shlykov I.L. Osseointegration of porous titanium modified by diamond-like carbon and carbon nitride. Diamond and Related Materials, 2012, vol. 22, pp. 128-135. DOI: 10.1016/j.diamond.2011.12.030. 6. Le Claire A. D. In: Diffusion in Solid Metals and Alloys, ed. by H. Mehrer, 1990, Group III, vol. 26, Landolt-Bцrnstein, Springer-Verlag, Berlin, p. 471.

7. Kidson G.V. In: Diffusion in Solid Metals and Alloys, ed. by H. Mehrer, 1990, Group III, vol. 26, Landolt-Bцrnstein, Springer-Verlag, Berlin, p. 504. 
8. Amsel G., Lanford W.A. Nuclear reaction techiques in materials analysys. Ann. Rev. Nucl. Part. Sci., 1984, vol. 34, pp. 435-460. DOI:10.1146/annurev.ns.34.120184.002251.

9. David D., Garcia E.A., Lucas X., Bŭranger G. Etude de la diffusion de l'oxygene dans le titane 6 oxyde entre $700^{\circ} \mathrm{c}$ et $950^{\circ} \mathrm{c}$. Journal of the Less Common Metals, 1979, vol. 65, No. 1, pp. 51-69. DOI: 10.1016/0022-5088(79)90152-8.

10. Kreuer K.D. On the complexity of proton conduction phenomena. Solid State Ionics, 2000, vol. 136-137 (1-2), pp. 149-160. DOI: 10.1016/S0167-2738(00)00301-5.

11. Kreuer K.D., Adams St., Mьnch W., Fuchs A., Klock U., Maier J. Proton conducting alkaline earth zirconates and titanates for high drain electrochemical applications. Solid State Ionics, 2001, vol. 145, pp. 295-306. DOI: 10.1016/S0167-2738(01)00953-5.

12. Kreuer K.D. Proton-Conducting Oxides. Annu. Rev. Mater. Res., 2003, vol. 33, pp. 333-359. DOI: 10.1146/annurev.matsci.33.022802.091825.

13. Pionke M., Mono T., Schweika W., Springer T., Schober H. Investigation of the hydrogen mobility in a mixed perovskite: $\mathrm{Ba}[\mathrm{Ca}(1+\mathrm{x}) / 3 \mathrm{Nb}(2-\mathrm{x}) / 3] \mathrm{O} 3-\mathrm{x} / 2$ by quasielastic neutron scattering. Solid State Ionics, 1997, vol. 97, pp. 497-504. DOI: 10.1016/S0167-2738(97)00077-5.

14. Karmonik Ch., Hempelmann R., Matzke Th., Springer T. Proton Diffusion in Strontium Cerate Ceramics studied by Quasielastic Neutron Scattering and Impedance Spectroscopy. Zeitschrift for Naturforschung A, 1995, vol. 50 (6), pp. 539-548. DOI: 10.1515/zna-1995-0605.

15. Qi Z., Volkl J., Lasser R., Wenzl H. Tritium diffusion in V, Nb and Ta. J. Phys. F: Met. Phys., 1983, vol. 13, pp. 2053-2062. DOI: 10.1088/0305-4608/13/10/015.

16. Kashlev Y.A. Three regimes of diffusion migration of hydrogen atoms in metals. Theor. Math. Phys., 2005, vol. 145, pp. 1590-1603. DOI: 10.1007/s11232-005-0185-8.

17. Sundell P.G., Wahnström G. Activation energies for quantum diffusion of hydrogen in metals and on metal surfaces using delocalized nuclei within the density-functional theory. Phys. Rev. Lett., 2004, vol. 92 (15), pp. 155901. DOI: 10.1103/PhysRevLett.92.155901.

18. Di Stefano D., Mrovec M., Elsдsser C. First-principles investigation of quantum mechanical effects on the diffusion of hydrogen in iron and nickel. Phys. Rev. B, 2015, vol. 92, pp. 224301. DOI: 10.1103/PhysRevB.92.224301.

19. Vykhodets V., Nefedova O., Kurennykh T., Obukhov S., Vykhodets Y. Quantum Diffusion of Deuterium in Sodium. J. Phys. Chem. A, 2019, vol. 123 (34), pp. 7536-7539. DOI: $10.1021 /$ acs.jpca.9b06231.

20. Kudo H., Kosaku Y., Ando Y., Higara M., Sekine T. Deuterium migration in titanium during deuteron irradiation observed by proton spectra of the $\mathrm{d}(\mathrm{d}, \mathrm{p}) \mathrm{t}$ reaction. Journal of Nuclear Materials, 1998, vols. 258-263, part 1, pp. 622-627. DOI: 10.1016/S0022-3115(98)00244-X.

21. Vykhodets Vladimir B., Jarvis Emily A.A., Kurennykh Tatiana E., Beketov Igor V., Obukhov Sviatoslav I., Samatov Oleg M., Medvedev Anatoly I., Davletshin Andrey E., Whyte Travis H. Inhomogeneous depletion of oxygen ions in oxide nanoparticles. Surface Science, 2016, vol. 644, pp. 141-147. DOI: 10.1016/j.susc.2015.10.011. 
Подана в журнал: 20.06.2021

УДк УДК 53.083.9

DOI: $10.17804 / 2410-9908.2021 .4 .006-014$

\author{
МЕТОД ЯДЕРНЫХ РЕАКЦИЙ IN SITU \\ В. Б. Выходец ${ }^{\text {a) }}$, Т. Е. Куренных ${ }^{\text {б)* }}$ \\ Федеральное государственное бюджетное учреждение науки \\ Институт физики металлов им. М.Н. Михеева Уральского отделения Российской академии наук, \\ д. 18, ул. С. Ковалевской, г Екатеринбург, Российская Федераџия \\ a) (iD http://orcid.org/0000-0001-9522-9147; \\ б) (iD http://orcid.org/0000-0001-9859-3374

\begin{abstract}
*Ответственный автор. Электронная почта: kurennykh@imp.uran.ru
Адрес для переписки: ул. С. Ковалевской, 18, г. Екатеринбург, Российская Федерация
\end{abstract} \\ Тел.: +7 (343) 378-38-46
}

Рассмотрены принципы методик ядерных реакций in situ, потребность в них в различных областях научных исследований и приведены несколько примеров применения этих методик. Показано, что методики ядерных реакций in situ эффективны при исследовании диффузии дейтерия в металлах при температурах ниже комнатной, диффузии дейтерия в протонпроводящих оксидах, квантовой диффузии дейтерия в металлах при криогенных температурах и химического состава оксидных нанопорошков при их нагреве в вакууме. Сформулированы перспективные направления использования методик ядерных реакций in situ.

Ключевые слова: метод ядерных реакций, дейтерий, металл, оксид, нанопорошок.

\title{
1. Введение
}

Первые исследования с помощью метода ядерных реакций (NRA) были выполнены в середине прошлого века. NRA получил широкое распространение и в течение нескольких десятков лет является одним из самых эффективных прямых методов определения концентраций изотопов легких элементов в твердых телах, их концентрационных профилей в образцах, применялся при исследовании диффузии легких элементов в твердых телах, химического состава тонких пленок и покрытий и в других областях физики, химии и материаловедения. Метод NRA предполагает облучение образцов на ускорителе и регистрацию продуктов ядерных реакций, протекающих во время облучения, между ускоренными частицами и содержащимися в образцах атомами легких элементов. Примерами таких реакций являются ${ }^{18} \mathrm{O}(\mathrm{p}, \alpha){ }^{15} \mathrm{~N}$ и ${ }^{16} \mathrm{O}\left(\mathrm{d}, \mathrm{p}_{1}\right){ }^{17}$. Они иллюстрируют изотопную чувствительность методики. Наиболее часто в исследованиях с помощью NRA используют протоны и дейтроны с энергиями до нескольких МэВ и регистрируют протоны и $\alpha$-частицы также с энергиями до нескольких МэВ.

Метод нашел широкое применение, поскольку его преимуществами были изотопная чувствительность, высокая точность при измерении концентраций легких элементов, высокая чувствительность при измерении низких концентраций (до $10^{-3}$ ат. \%,), возможность измерения концентрационных профилей изотопов без разрушения образца с разрешением по глубине от 0,1 мкм до глубины в образце около 2 мкм, локальность измерений по поверхности образца на уровне 0,1 мм, абсолютный характер измерений концентраций (это обусловлено тем, что сечения ядерных реакций являются их фундаментальными характеристиками). Распространению NRA способствовало несколько обстоятельств. Во-первых, атомы легких элементов даже при очень низких концентрациях сильно влияют на свойство металлов, они входят в качестве основных компонентов в состав оксидов, нитридов, карбидов и других со- 
единений. Во-вторых, измерение концентраций легких элементов было большой проблемой для существующих методов исследования элементного и изотопного состава твердых тел, дополнительно к этому для легких элементов практически отсутствуют радиоактивные изотопы с характеристиками, приемлемыми для диффузионных исследований. Научно-методической базой методики NRA являются многочисленные результаты по сечениям ядерных реакций, тормозным способностям элементов, длинам пробега ускоренных ионов в материалах и другая информация $[1,2]$

Среди важных результатов, полученных с помощью NRA, можно отметить данные по коэффициентам диффузии атомов легких элементов в металлах и оксидах, составу пленок и покрытий, металлургии титановых сплавов, синтезу оксидных нанопорошков и др. [3-8]. Основным конкурентом методики NRA в течение многих лет была вторичная ионная массспектрометрия.

В последние годы прогресс в применении некоторых методик в измерении концентраций легких элементов в твердых телах был значительным. Прежде всего нужно отметить появление спектральных приборов компании LECO, с помощью которых концентрации кислорода, водорода и азота в твердых телах могут быть определены с чувствительностью до $10^{-6}$ aт. \%, что на несколько порядков превосходит возможности NRA. В то же время преимуществами NRA перед спектрометрами LECO остаются изотопная чувствительность и высокая локальность измерений по поверхности и глубине в образце. Отметим, что в прибоpax LECO необходимая масса образца для анализа составляет 1 г. В последнее время вырос интерес к разработке материалов для литийсодержащих аккумуляторов и NRA применяется в этих исследованиях, поскольку превосходит возможности других методик для измерения концентраций лития. В данном случае речь идет не о прогрессе в использовании NRA, a о eе традиционном применении. Новым же подходом, который имел место при развитии методик NRA, является их использование в режиме in situ, и настоящая работа ориентирована на анализ первых результатов в этом направлении.

\section{2. Причины для применения методик NRA in situ}

В нескольких работах последних лет обозначились новые тенденции в развитии метода NRA. Они направлены на проведение измерения концентрационных профилей изотопов легких элементов непосредственно во время процесса - предмета научного исследования. Это может быть, например измерение концентрационных профилей атомов во время диффузии или в процессе формирования тех или иных сегрегаций или определение состава образца по легким элементам при протекании фазовых превращений. Существует несколько объективных причин для применения методик NRA in situ, приведем несколько примеров.

Для атомов самого легкого элемента водорода для некоторых типов твердых тел, в частности протонпроводящих оксидов, характерны высокие значения коэффициентов диффузии водорода даже при комнатной температуре. По этой причине для таких случаев не приемлемы традиционные постановки диффузионных экспериментов, при которых диффузионные отжиги образцов проводят при высоких или повышенных температурах, а измерение концентрационных профилей - при комнатной температуре. В связи с этим в протонпроводящих оксидах для получения данных по коэффициентам диффузии водорода применяли непрямые методы, например протонную проводимость [9-13]. Использование непрямых методов для измерения коэффициентов диффузии водорода в твердых телах получило широкое распространение, однако в любом случае применение для этой цели прямых методов, основанных на диффузионных уравнениях Фика, является актуальной задачей. В работе мы не останавливаемся подробно на роли прямых и непрямых методов исследования, ограничимся ремаркой, что для получения информации о механизмах миграции атомов в твердых телах необходимо применение тех и других подходов. 
Следующий пример также связан с диффузией, причем он имеет фундаментальное значение. Существуют всего два механизма миграции атомов в твердых телах: надбарьерные прыжки атомов (классический механизм) и туннелирование (квантовый). Почти все опытные данные по диффузии относятся к классическому механизму миграции, однако в случае атомов легких элементов большой практический и теоретический интерес представляют диффузионные данные для квантового механизма миграции [14-17]. Они актуальны для теорий диффузии, квантовой химии, низкотемпературных технологий и астрофизики, прежде всего для водорода в связи с его участием во многих природных и технологических процессах. Поскольку квантовую диффузию наблюдают только при температурах ниже комнатной, в этих случаях, очевидно, не может быть применена традиционная схема диффузионных экспериментов, при которой измерение диффузионных профилей атомов проводится при комнатной температуре. Таким образом, приходим к заключению, что в случае изотопов водорода при температурах, близких или ниже комнатной, актуальны постановки диффузионных исследований в режиме in situ.

Применение методики NRA in situ может быть актуально не только для измерения концентрационных профилей водорода, а также для такого класса материалов как оксидные и другие нанопорошки и наноразмерные пленки. Дело в том, что химический состав наночастиц и тонких пленок из-за их большой удельной поверхности и высокой химической активности сильно изменяется при хранении на воздухе и исследование состава наночастиц и пленок по водороду, кислороду и другим легким элементам с использованием методики NRA целесообразно проводить в условиях, когда их пребывание на воздухе в течение всего цикла исследований исключено. Мы рассмотрели некоторые частные примеры и можно констатировать, что они показывают высокую потребность в развитии методик NRA in situ для материаловедения, электрохимии, наноматериалов, квантовой диффузии в металлах и других научных направлений. Далее в работе рассмотрены основные принципы методик NRA in situ, а также некоторые первые научные результаты, полученные с их помощью.

\section{3. Принципы методик NRA in situ}

В литературе упоминаются следующие принципы, которые могут быть использованы при разработке методик NRA in situ. Первый из них связан с температурными условиями экспериментов при использовании методики NRA, которые обычно проводят при комнатной температуре. В такой постановке предметом исследований являются концентрационные профили атомов, сформированные при высокотемпературных процессах, например синтезе, выплавке, диффузионном отжиге или хранении образцов в тех или иных условиях. В таких случаях речь не идет о режимах in situ и необходимым условием их реализации является возможность проведения исследований с использованием NRA в широком интервале температур. В целом это традиционная задача для многих методик, например для электронной и оптической микроскопии, и в случае NRA можно отметить только наличие естественной верхней границы для температуры образца около $500{ }^{\circ} \mathrm{C}$, более высокие температуры противопоказаны применению кремниевых поверхностно-барьерных детекторов, используемых для регистрации продуктов ядерных реакций. В работах $[18,19]$ для нагрева и охлаждения образцов использовали резистивную печь и проточный жидкий азот, в этом случае температура кипения жидкого азота была нижней границей диффузионных исследований.

При диффузионных исследованиях важным вопросом, связанным с температурными условиями экспериментов, является точность поддержания и измерения температуры при проведении изотермических отжигов в вакуумной камере ускорительной установки. Эта задача не вполне тривиальна, поскольку поддержание необходимого температурного режима осуществляется во время облучения образцов, подачи в камеру ускорительной установки жидкого азота и эксплуатации резистивной печи. Задача носит многопараметрический характер, поскольку функционирование каждого из перечисленных факторов 
приводит к колебаниям температуры образца. Задача поддержания постоянной температуры образца в вакуумной камере ускорительной установки не может быть решена при помощи традиционных регуляторов, здесь необходимы специальные подходы. Можно констатировать, что удовлетворительное решение задачи было найдено, например в работе [18], где температуру образца при диффузионных отжигах поддерживали постоянной и измеряли с точностью $\pm 1 \mathrm{~K}$. Высокая точность стабилизации температуры в упомянутых работах достигалась за счет того, что колебания мощности нагревателя, тока пучка и тепловых потоков в камере ускорителя при облучении автоматически компенсировались изменениями теплового потока от пластины с образцом к массивному теплообменнику, охлаждаемому проточным жидким азотом. Кроме того, в этих работах предпринимались независимые меры для стабилизации тока пучка ускорителя, потока жидкого азота через теплообменник в камере ускорительной установки и мощности резистивного нагревателя. Таким образом, для методики NRA in situ может быть обеспечен температурный интервал исследований примерно от 80 до 800 К с точностью поддержания температуры образца при изотермических отжигах, достаточной для диффузионных исследований.

Второй принцип методики NRA in situ заключается в использовании ионов пучка ускорителя для двух целей: легирования ими образца и проведения ядерной реакции. Это положение удобно проиллюстрируем на частном примере. При облучении дейтронами происходит их имплантация в образец, в результате ионы дейтерия распределяют в образце в соответствии с теми или иными физико-химическими процессами. Это могут быть, например, фазовые превращения, формирование диффузионных распределений, формирование сегрегаций атомов на примесях, границах образца, дефектах решетки и т. д. Параллельно с этими процессами протекает ядерная реакция ${ }^{2} \mathrm{H}(\mathrm{d}, \mathrm{p})^{3} \mathrm{H}$ между имплантированными атомами дейтерия ${ }^{2} \mathrm{H}$ и ионами пучка $\mathrm{d}$ с выходом высокоэнергетических протонов р, спектры которых регистрируются с помощью полупроводниковых детекторов. Ясно, что с помощью такой схемы эксперимента можно in situ получить информацию о физико-химических процессах, происходящих с атомами дейтерия в материале. Этот принцип организации эксперимента может представлять интерес по нескольким причинам. Во-первых, он сочетает достоинства методик имплантации и NRA, поскольку позволяет получать информацию о поведении водорода, например диффузии и фазовых превращениях в материалах, в которые водород нельзя ввести с помощью отжигов. В частности, это могут быть металлы, в которых водород практически нерастворим или которые разрушаются при отжигах в агрессивных водородосодержащих средах. Во-вторых, у такого подхода есть важные преимущества перед традиционными постановками эксперимента. Они обусловливают расширение диапазона измеряемых значений коэффициентов диффузии водорода или интервала температур, для которого могут быть получены результаты по тому или иному явлению. Эти вопросы нуждаются в специальных исследованиях, но в любом случае преимущества подходов, связанных с применением методик NRA in situ, очевидны, поскольку имплантация возможна при любых температурах образца, а другие способы ввода атомов водорода в материалы образца обычно требуют повышенных температур.

Мы привели частный пример использования методики NRA in situ, когда для целей легирования образца и проведения ядерной реакции используются ионы дейтерия. Вероятно, аналогичные варианты могут быть предложены для других ускоренных частиц.

\section{4. Примеры и перспективы применения методик NRA in situ}

Примеры применения методик NRA in situ пока не многочисленны, и мы остановимся только на двух результатах. В работе [20] было проведено исследование отклонений от стехиометрии в наночастицах YSZ10 - кубического диоксида циркония, допированного 10 мол. \% иттрия. С помощью NRA и изотопных методов было установлено, что на этапе высокотем- 
пературного синтеза нанопорошка, который проводился с помощью технологии лазерного испарения керамической мишени, имел место сильный кислородный дефицит в поверхностном атомном слое наночастиц. Этот эффект был прогнозируем теорией. Однако при комнатной температуре порошка отклонений от стехиометрии практически не было, т. е. при охлаждении на воздухе происходило окисление наночастиц. Установить механизм этого окисления не представлялось возможным, поскольку пребывание порошка на воздухе и его взаимодействие с окружающей атмосферой было неизбежным этапом при работе с порошками. В связи с этим в работе [20] была применена методика NRA in situ, а именно, проводилось измерение концентрации кислорода в порошке с помощью методик NRA во время нагрева порошка непосредственно в вакуумной камере ускорительной установки. По результатам этого исследования удалось установить, что термодинамически равновесный кислородный дефицит в кристаллической решетке наночастицы сохраняется до комнатной температуры, а изменение состава порошка при охлаждении на воздухе обусловлено процессами абсорбции и хемосорбции водяных паров, углекислого газа и кислорода.

Результаты этого эксперимента показывают, что методика NRA in situ имеет перспективы не только в аналогичных применениях, но и для получения данных по температурной зависимости коэффициентов диффузии легких элементов при температурах, которые незначительно выше комнатной. Объектами исследования в этих случаях могут быть, например, оксидные нанопорошки, подвергнутые предварительному стабилизирующему отжигу в атмосфере кислорода.

Другой пример использования методики NRA in situ связан с исследованием температурной зависимости коэффициентов диффузии дейтерия в щелочном металле натрии [18]. Отметим, что в литературе отсутствовали опытные данные по диффузии изотопов водорода в щелочных металлах. Такое положение связано с тем, что они являются неудобными объектами для диффузионных исследований. Осложняющими факторами являются, в частности, образование гидридов при отжиге щелочных металлов в водороде и их окисление даже при низких парциальных давлениях кислорода. Эта же ситуация имеет место со многими лантаноидами и щелочноземельными металлами, для них также характерно почти полное отсутствие экспериментальных данных по диффузии водорода [6].

Задача измерения коэффициентов диффузии дейтерия в натрии решалась с помощью подхода, при использовании которого ионная имплантация, диффузионный отжиг и измерение диффузионного распределения атомов дейтерия осуществлялись одновременно. Измерение концентрационного профиля ионов дейтерия проводилось с помощью методики NRA, использовалась реакция ${ }^{2} \mathrm{H}(\mathrm{d}, \mathrm{p})^{3} \mathrm{H}$. Пучок дейтронов использовался как для имплантации ионов дейтерия в образец, так и для измерений концентрации дейтерия в образце. Идея такого эксперимента впервые была предложена в работе [19]. Эта методика получила название nuclear reaction analysis on-line (NRAOL), она является вариантом методики NRA in situ. Дополнительно к этому в работе [18] был решен вопрос о применении методики NRA для исследования материалов, которые легко окисляются на воздухе и в состоянии поставки хранятся в стеклянных ампулах, керосине и других изолирующих средах. Для этого использовался герметичный транспортный контейнер. Ампулы с натрием вскрывали в боксе в атмосфере аргона с содержанием кислорода и водяного пара не более 0,5 ppm. В боксе натрий переносили в герметичный транспортный контейнер, его переносили в камеру ускорительной установки и вскрывали только после получения в камере высокого вакуума.

В работе [18] данные по диффузии дейтерия в натрии были получены в интервале температур от 110 до $240 \mathrm{~K}$ в диапазоне значений коэффициентов диффузии от $10^{-16}$ до $10^{-12} \mathrm{~m}^{2} / \mathrm{c}$. Это на настоящее время самый существенный результат, полученный с помощью метода NRA in situ. Он свидетельствует о том, что при помощи этой методики могут быть ликвидированы «белые пятна» в опытных данных по диффузии водорода в лантаноидах, щелочных, щелочноземельных и других металлах, которые не могут быть подвергнуты диффузионному отжигу в водородосодержащих средах. Также эти результаты показывают, что при использо- 
вании метода NRA in situ могут быть получены данные по диффузии дейтерия во всех материалах при температурах ниже комнатной.

\section{5. Заключение}

Показано, что для получения экспериментальных данных по диффузии легких элементов в твердых телах во многих случаях целесообразно применять ускорительную методику ядерных реакций in situ. Одним из возможных условий ее реализации является нагрев, охлаждение и изотермический отжиг образца в вакуумной камере ускорительной установки одновременно с его облучением и регистрацией продуктов ядерных реакций. Другим вариантом может быть использование пучка ускорителя одновременно для измерения концентрационных профилей легкого элемента в образце и его легирования. Приведены примеры применения методики ядерных реакций in situ, впервые выполнены исследования температурной зависимости коэффициентов диффузии водорода в щелочном металле и установлена природа отклонений от стехиометрии в оксидных наночастицах.

\section{Благодарность}

Работа выполнена в рамках государственного задания МИНОБРНАУКИ России (тема «Функиия», № АAАA-A19-119012990095-0)

\section{Литература}

1. The Stopping and Ranges of Ions in Matter (SRIM-2013): collection of software packages. URL: http://www.srim.org.

2. Ziegler J. F., Biersack J. P., Littmark U. The Stopping and Ranges of Ions in Solids. - N.Y. : Pergamon Press, 1984. - Vol. 1.

3. The interrelation between structure and mechanical properties of $\mathrm{CN} x$ films $(0 \leq \mathrm{x} \leq 0.5)$, deposited by arc sputtering of graphite / A. P. Rubshtein, I. Sh Trakhtenberg, E. G. Volkova, A. B. Vladimirov, A. G. Gontar, K. Uemura // Diamond and Related Materials. - 2005. - Vol. 14. P. 1820-1823. - DOI: 10.1016/J.DIAMOND.2005.07.016.

4. Mechanical properties of $\mathrm{CN} x$ coatings obtained by carbon arc sputtering / I. Sh. Trakhtenberg, A. B. Vladimirov, A. P. Rubstein, V. A. Yugov, V. B. Vykhodets, T. E. Kurennykh, A. G. Gontar, V. N. Tkach, S. N. Dub, and K. Uemura // Journal of Superhard Materials. - 2007. - Vol. 29, No. 3. - P. 138-141. - DOI: 10.3103/S1063457607030045.

5. Osseointegration of porous titanium modified by diamond-like carbon and carbon nitride / A. P. Rubstein, E. B. Makarova, I. Sh. Trakhtenberg, I. P. Kudryavtseva, D. G. Bliznets, Yu. I. Philippov, I. L. Shlykov // Diamond and Related Materials. - 2012. - Vol. 22. - P. 128-135. DOI: 10.1016/j.diamond.2011.12.030.

6. Diffusion in Solid Metals and Alloys / ed. by H. Mehrer / A. D Le Claire. - 1990. Group III, vol. 26. - Berlin : Landolt-Bцrnstein, Springer-Verlag, 1990. - P. 471.

7. Diffusion in Solid Metals and Alloys / ed. by H. Mehrer / G. V. Kidson. - 1990. - Group III, vol. 26. - Berlin : Landolt-Bцrnstein, Springer-Verlag, 1990 - P. 504.

8. Amsel G., Lanford W. A. Nuclear reaction techiques in materials analysys // Ann. Rev. Nucl. Part. Sci. - 1984. - Vol. 34. - P. 435-460. - DOI: 10.1146/annurev.ns.34.120184.002251.

9. Etude de la diffusion de l'oxygene dans le titane 6 oxyde entre $700^{\circ} \mathrm{c}$ et $950{ }^{\circ} \mathrm{c} / \mathrm{D}$. David, E. A. Garcia, X. Lucas, G. Bŭranger // Journal of the Less Common Metals. - 1979. - Vol. 65, No. 1. - P. 51-69. - DOI: 10.1016/0022-5088(79)90152-8.

10. Kreuer K. D. On the complexity of proton conduction phenomena // Solid State Ionics. 2000. - Vol. 136-137 (1-2). - P. 149-160. - DOI: 10.1016/S0167-2738(00)00301-5.

11. Proton conducting alkaline earth zirconates and titanates for high drain electrochemical applications / K. D. Kreuer, St. Adams, W. Mьnch, A. Fuchs, U. Klock, J. Maier // Solid State Ionics. 2001. - Vol. 145. - P. 295-306. - DOI: 10.1016/S0167-2738(01)00953-5. 
12. Kreuer K. D. Proton-Conducting Oxides // Annu. Rev. Mater. Res. - 2003. - Vol. 33. P. 333-359. - DOI: 10.1146/annurev.matsci.33.022802.091825.

13. Investigation of the hydrogen mobility in a mixed perovskite: $\mathrm{Ba}[\mathrm{Ca}(1+\mathrm{x}) / 3 \mathrm{Nb}(2-\mathrm{x}) / 3] \mathrm{O} 3-\mathrm{x} / 2$ by quasielastic neutron scattering / M. Pionke, T. Mono, W. Schweika, T. Springer, H. Schober // Solid State Ionics. - 1997. - Vol. 97. - P. 497-504. - DOI: 10.1016/S0167-2738(97)00077-5.

14. Proton Diffusion in Strontium Cerate Ceramics studied by Quasielastic Neutron Scattering and Impedance Spectroscopy / Ch. Karmonik, R. Hempelmann, Th. Matzke, T. Springer // Zeitschrift for Naturforschung A. - 1995. - Vol. 50 (6). - P. 539-548. - DOI: 10.1515/zna-1995-0605.

15. Tritium diffusion in V, Nb and Ta / Z. Qi, J. Volkl, R. Lasser, H. Wenzl // J. Phys. F: Met. Phys. - 1983. - Vol. 13. - P. 2053-2062. - DOI: 10.1088/0305-4608/13/10/015.

16. Kashlev Y. A. Three regimes of diffusion migration of hydrogen atoms in metals // Theor. Math. Phys. - 2005. - Vol. 145. - P. 1590-1603. - DOI: 10.1007/s11232-005-0185-8.

17. Sundell P. G., Wahnström G. Activation energies for quantum diffusion of hydrogen in metals and on metal surfaces using delocalized nuclei within the density-functional theory // Phys. Rev. Lett. - 2004. - Vol. 92 (15). - P. 155901. - DOI: 10.1103/PhysRevLett.92.155901.

18. Di Stefano D., Mrovec M., Elsдsser C. First-principles investigation of quantum mechanical effects on the diffusion of hydrogen in iron and nickel // Phys. Rev. B. - 2015. - Vol. 92. P. 224301. - DOI: 10.1103/PhysRevB.92.224301.

19. Quantum Diffusion of Deuterium in Sodium / V. Vykhodets, O. Nefedova, T. Kurennykh, S. Obukhov, Y. Vykhodets // J. Phys. Chem. A. - 2019. - Vol. 123. - P. 7536-7539. DOI: 10.1021/acs.jpca.9b06231.

20. Deuterium migration in titanium during deuteron irradiation observed by proton spectra of the d(d,p)t reaction / H. Kudo, Y. Kosaku, Y. Ando, M. Higara, T. Sekine // Journal of Nuclear Materials. - 1998. - Vols. 258-263 (1). - P. 622-627. - DOI: 10.1016/S0022-3115(98)00244-X.

21. Inhomogeneous depletion of oxygen ions in oxide nanoparticles / Vladimir B. Vykhodets, Emily A. A. Jarvis, Tatiana E. Kurennykh, Igor V. Beketov, Sviatoslav I. Obukhov, Oleg M. Samatov, Anatoly I. Medvedev, Andrey E. Davletshin, Travis H. Whyte // Surface Science. - 2016. Vol. 644. - P. 141-147. - DOI: 10.1016/j.susc.2015.10.011. 